\title{
EXTRACELLULAR CURRENT FLOW AND THE SITE OF TRANSDUCTION BY VERTEBRATE HAIR CELLS ${ }^{1}$
}

\author{
A. J. HUDSPETH ${ }^{2}$
}

Beckman Laboratories of Behavioral Biology, Division of Biology 216-76, California Institute of Technology, Pasadena, California 91125

Received July 23, 1981; Accepted September 28, 1981

\begin{abstract}
The transduction process of a vertebrate hair cell commences with the application of mechanical stimuli to the hair bundle, a cluster of microvillous stereocilia and single, axonemal kinocilium. In an effort to determine where within the hair bundle transduction occurs, I have measured extracellular potentials around the hair bundles of mechanically stimulated hair cells from the bullfrog's sacculus. Stimulus-dependent signals up to $17 \mu \mathrm{V}$ in peak-to-peak amplitude have been found. These appear to be due to the flow of transduction current on the basis of their amplitude, phase, dependence on stimulus size and orientation, proportionality to membrane potential, and sensitivity to an ototoxic antibiotic. The responses are consistently larger near the top of the hair bundle than at its base, suggesting that the transduction apparatus lies at or near the distal ends of the stereocilia.
\end{abstract}

The mechanically sensitive component of hair cells, the hair bundle, comprises 30 to 200 clavate, microvillous stereocilia protruding from the hair cell's apex in a hexagonal array. The stereocilia vary monotonically in length from one edge of the hair bundle to the other so that the hair bundle appears to be truncated obliquely at its top. In addition, all vertebrate hair cells possess a solitary true cilium, the kinocilium, at least during ontogeny. This cilium occurs at the edge of the hair bundle where the stereocilia are longest.

Sounds, accelerations, vibrations, and water movements act through mechanical linkages of varying complexity to deflect the distal tips of hair bundles in the various acoustico-lateralis organs. The stereocilia, which at least grossly act as stiff rods (Engström et al., 1962), pivot at their bases; the resulting displacement of the hair bundle's tip produces a membrane conductance change that results in a receptor potential (Hudspeth and Corey, 1977).

\footnotetext{
' This research was supported by National Institutes of Health Grant NS-13154 and by grants from the Ann Peppers, William Randolph Hearst, and Pew Foundations. I thank Dr. G. Green for solving the short-cable equations for harmonic stimuli, Mr. R. Jacobs for assistance with histology and computations, and Dr. D. P. Corey for helpful discussions. Ms. P. Brown kindly typed the manuscript, and Drs. R. A. Eatock and J. H. R. Maunsell provided helpful criticism as well as aid with signal averaging.

${ }^{2}$ To whom correspondence should be addressed at Division of Biology 216-76, California Institute of Technology, 1201 East California Boulevard, Pasadena, CA 91125.
}

The complex geometry of the hair bundle and the small displacements to which it responds (Hudspeth and Corey, 1977) make it difficult to determine which of several distinct mechanisms is responsible for transduction. It is possible that deflection of the stereocilia opens ionic channels along their strained lengths or at their basal pivots (Flock et al., 1977). The stereocilia might act as levers, applying force to a sensitive element at the base of the kinocilium or elsewhere on the apical cellular surface (Engström et al., 1962; Hillman, 1972). Displacement of the hair bundle could alter the separation among stereocilia and thus affect the exposed area of ion-permeable membrane (Malcolm, 1974). Another possibility is that interactions at the distal tips of the stereocilia produce a response there.

The present study is based on the hypothesis that the site at which transduction current enters the hair cell is physically close to-if not identical with - the transduction element at which the response to mechanical stimulation originates. Measurement of extracellular potentials around responding hair cells indicates a predominance of current flow into the distal portion of the hair bundle, suggesting that the transduction process is localized near the tips of the stereocilia.

\section{Materials and Methods}

Experimental preparation. Saccular maculae, dissected from bullfrogs (Rana catesbeiana) 90 to $150 \mathrm{~mm}$ in snout-vent length, were maintained in a saline solution consisting of $120 \mathrm{~mm} \mathrm{Na}{ }^{+}, 2 \mathrm{mM} \mathrm{K}{ }^{+}, 4 \mathrm{mM} \mathrm{Ca}^{2+}, 128 \mathrm{mM}$ $\mathrm{Cl}^{-}, 3 \mathrm{~mm}$ D-glucose, and $5 \mathrm{~mm}$ 4-(2-hydroxyethyl)-1- 
piperazine-ethanesulfonic acid (HEPES), $\mathrm{pH} 7.2$ to 7.3. Subtilopeptidase A was used to loosen the otolithic membrane and facilitate its removal by dissection (Corey and Hudspeth, 1979a). The macula was mounted flat against a glass coverslip at the bottom of a $0.5-\mathrm{ml}$ experimental chamber placed on the fixed stage of a Zeiss WL microscope (Karl Zeiss, Oberkochen, German Federal Republic). The saline solution was exchanged at roughly $10-\mathrm{min}$ intervals to compensate for evaporation; heat filters were used in the illumination system to maintain the preparation's temperature at $22^{\circ} \mathrm{C}$.

The preparation was viewed through a $\times 40$, water immersion objective lens of numerical aperture 0.75 ; the total magnification of the Nomarski differential interference contrast system was $\times 1000$. A calibrated eyepiece micrometer was employed to estimate horizontal distance to about $\pm 0.1 \mu \mathrm{m}$.

Dimensions of hair bundles. The positions of components of the hair bundle and of recording electrodes are described in terms of a right-handed, cartesian coordinate system with its origin at the base of the kinocilium (Shotwell et al., 1981). The $x$ axis bisects the hair bundle (Fig. $1 A$ ) along its axis of bilateral symmetry, with the positive direction that in which the kinucilium lies with respect to the stereocilia. The $y$ axis is perpendicular to the $x$ axis and in the plane of the epithelial surface, while the $z$ axis corresponds to height above this plane. The average dimensions of the constituents of hair bundles in large hair cells from the sacculus were established by analysis of living preparations and by scanning and freeze-fracture transmission electron microscopy (A. J. Hudspeth and R. Jacobs, unpublished observations). The positions of the distal tips and insertions of the stereocilia and kinocilium, which were employed in modeling the results of the present study, were calculated from these data with appropriate corrections for shrinkage of the fixed material.

Preparations used in scanning electron microscopy were osmium fixed, dehydrated, critical-point dried, and sputter coated as described previously (Hudspeth and Jacobs, 1979). Material for freeze-fracture electron microscopy was fixed in $200 \mathrm{~mm}$ glutaraldehyde and $5 \mathrm{~mm}$ $\mathrm{CaCl}_{2}$ buffered to $\mathrm{pH} 7.25$ with $80 \mathrm{~mm}$ sodium cacodylate. After equilibration with $3.1 \mathrm{M}$ glycerol, tissue was frozen in monochlorodifluoromethane at its melting point, fractured (model BA360M, Balzers Aktiengesellschaft, Fürstentum, Liechtenstein), and replicated with a platinum sputter-coating gun.

Stimulation. Mechanical stimuli were applied to hair bundles by a blunt, horizontally mounted, $0.5-\mu \mathrm{m}$-diameter glass probe placed tangentially against the short edge of the hair bundle about $2 \mu \mathrm{m}$ above the epithelial surface. This stimulation technique was chosen because it provided better access by the recording electrode to sites of interest than did application of stimuli either to the tip of the hair bundle (Hudspeth and Corey, 1977) or to the junction of the kinocilium with the adjacent stereocilia (Corey and Hudspeth, 1979a). The probe was pushed against the hair bundle with a two-dimensional piezoelectric stimulator (Corey and Hudspeth, 1980); a $25-\mathrm{Hz}$, sinusoidal displacement was employed ordinarily. The amplitude of the displacement was adjusted to pro- duce a $1.0-\mu \mathrm{m}$ oscillation of the hair bundle at the level of the kinocilium's bulbous tip, $6.7 \mu \mathrm{m}$ above the cellular surface. The stimulation frequency chosen was slow enough for adequate assessment of probe and hair bundle motion, fast enough to allow collection of data in a reasonable time, and sufficiently far from the power line frequency $(60 \mathrm{~Hz})$ and its subharmonics to minimize interference from that source. The relatively large stimulus amplitude was chosen to ensure constantly nearmaximal responses despite some drift of the microscope stage and stimulus probe during the long recording period.

Recording procedure. Glass microelectrodes with short tapers $(\sim 5 \mathrm{~mm})$ and $0.4-\mu \mathrm{m}$ tips were pulled at low heat and tension settings on conventional microelectrode pullers. The electrodes were filled with saline solution identical to that in the experimental chamber; this relatively low conductance solution yielded electrode resistances of about 35 megohms. Although filling with $3 \mathbf{~ M}$ $\mathrm{KCl}$ substantially lowered electrode resistance and thus noise, the tip diffusion potential which ensued proved to be modulated by the stirring effect of the stimulus probe, producing an artifactual potential at the same frequency as the physiological signal sought.

Recordings were made principally from large hair cells near the margin of the saccular macula opposite the nerve's insertion. These cells correspond to those used in previous studies of intracellular receptor potentials (Hudspeth and Corey, 1977). After preliminary experiments were completed, data were obtained from 289 potential measurements in 24 hair cells from six animals.

Electrodes were positioned with a three-dimensional piezoelectric micromanipulator (Corey and Hudspeth, 1980). The circuitry controlling the manipulator was calibrated to give electrical monitor outputs of $0.6 \mathrm{~V}$ per $\mu \mathrm{m}$ of electrode movement along each axis. In most experiments, the electrode approached the hair bundle horizontally and directly from the side, parallel with the cellular $y$ axis.

The epithelial surface was located by situating the recording electrode aside the hair bundle to be studied and lowering it until a slight dimpling in the surface of a supporting cell was noted. With this position as a reference, the height of the electrode above the epithelial surface-the $z$ axis coordinate-then was indicated by the $z$ axis monitor signal. The electrode tip was positioned in the $x-y$ plane by locating it relative to landmarks on the hair bundle and approximately $0.5 \mu \mathrm{m}$ outside of the volume occupied by the vibrating hair bundle. Recording sites on the beveled top surface of the hair bundle were defined by locating the electrode tip appropriately in the $x$ and $y$ dimensions and then lowering it until it was just above the hair bundle. Lowering the electrode too far was reliably signaled by a diminution in the recorded signal due to impediment of the hair bundle's motion by the recording electrode. The uncertainty in electrode tip position at each recording site was estimated at $\pm 0.2 \mu \mathrm{m}$ along each axis.

A complete scan of the potential about a hair bundle comprised a total of 21 measurements. The recording sequence in half of the scans corresponded to sites (Fig. 1B) $16,17,18,5,10,4,9,3,8,13,2,7,12,14,1,6,11,15$, 


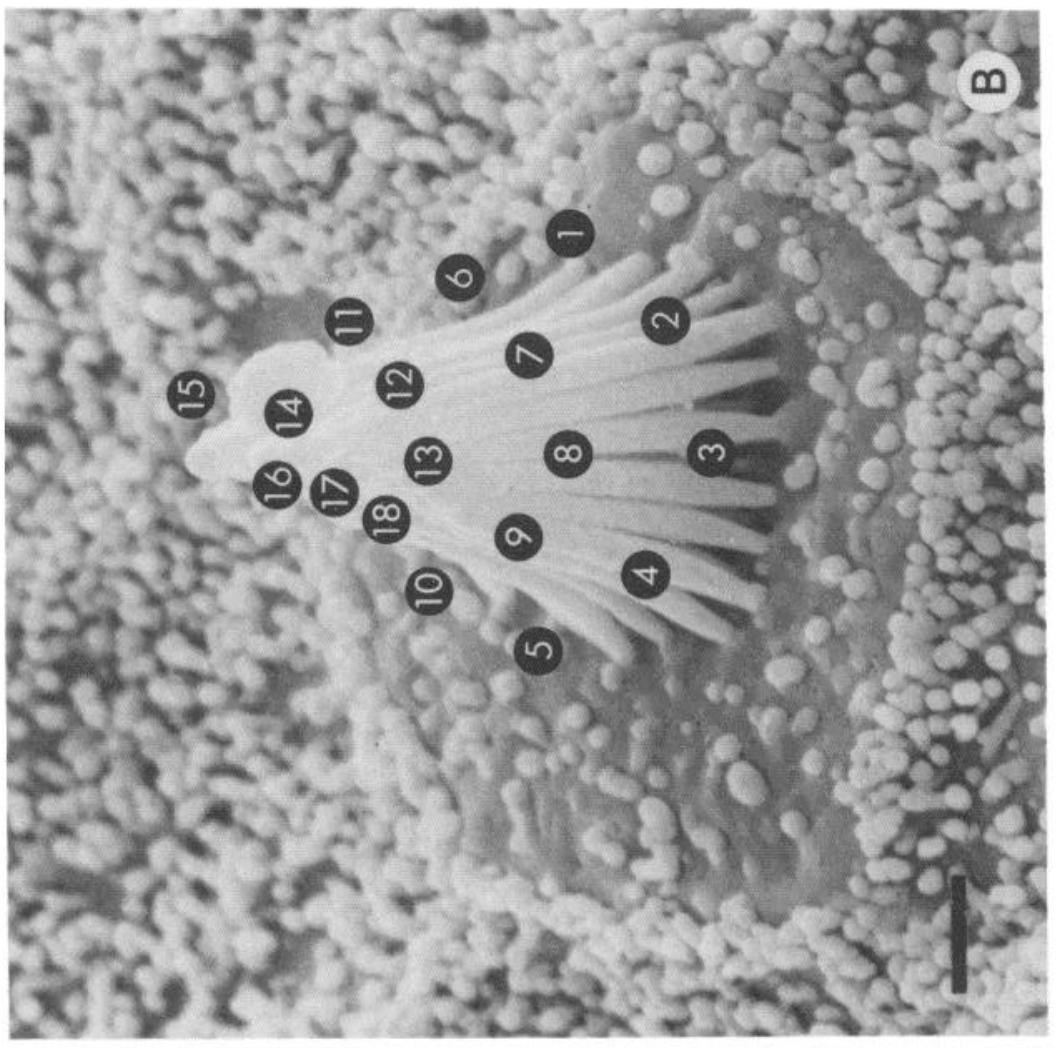

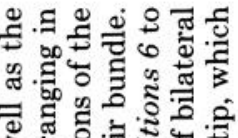
34.0 .0

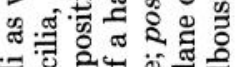
零 क्षे

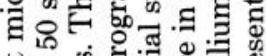
क का की क्षे สี 年

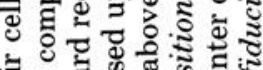

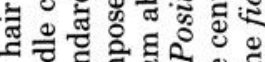

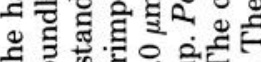

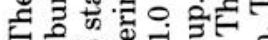
$\checkmark$ :

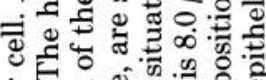

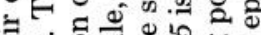
娄. สี

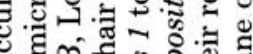
हn สำ.

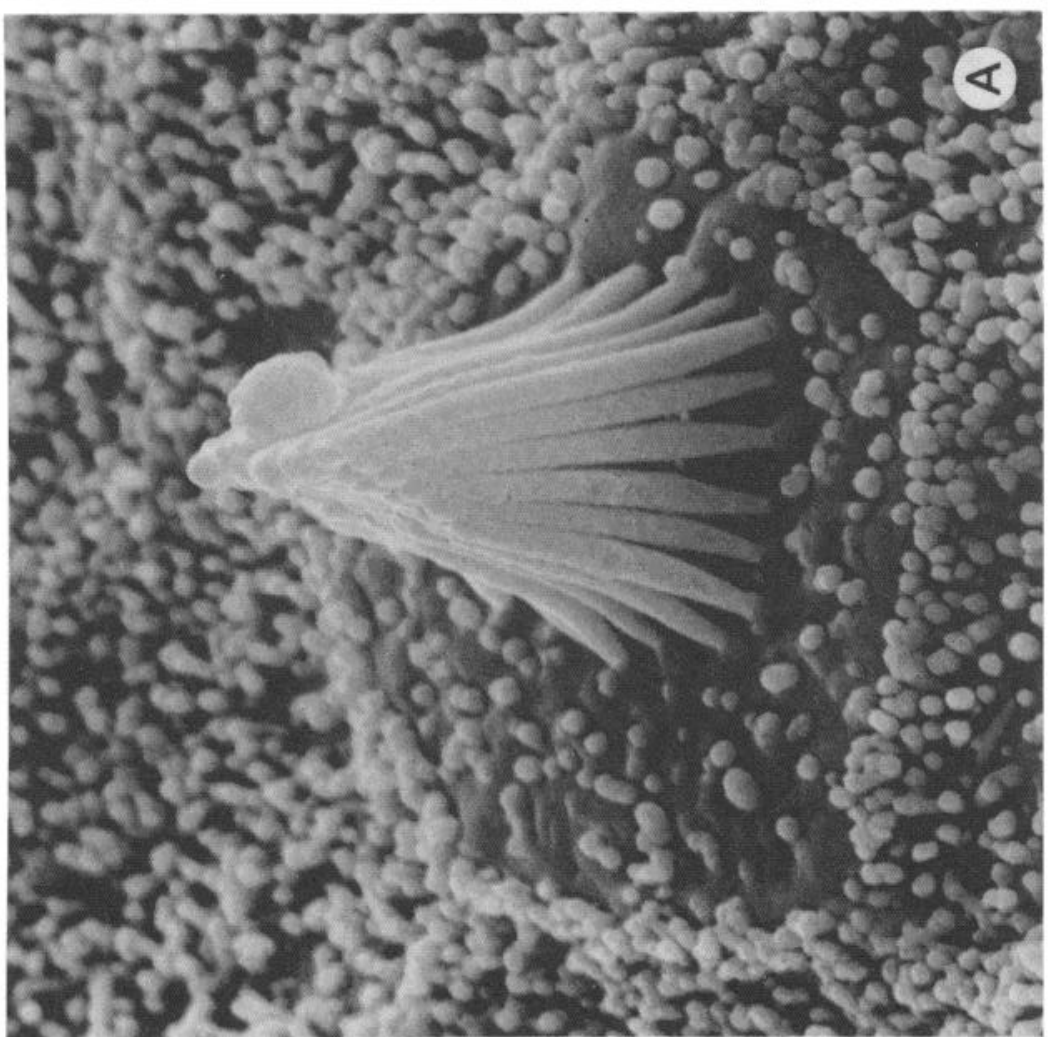

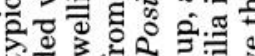
क त क्ष

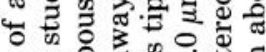
\& 范

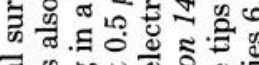
ฐొ ส.

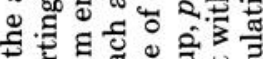
范茟

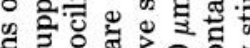
굴.

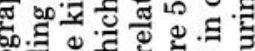
월

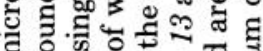
a

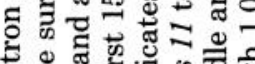

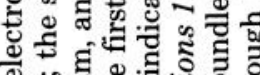

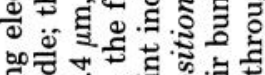

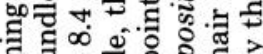

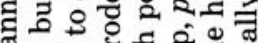
땐

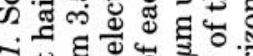

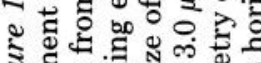

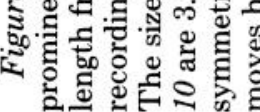


18,17 , and 16 ; alternate scans were done in the opposite order. As reflected in the data of Table I, a restricted series of measurements was made in many instances. Finally, controls for electrical pickup or hydrodynamic potentials were done by removing the stimulus probe from the hair bundle and allowing it to oscillate less than $0.5 \mu \mathrm{m}$ from the electrode tip.

Because each measurement required 20 to $30 \mathrm{sec}$, a complete scan lasted over $10 \mathrm{~min}$. The repeat measurements at positions 16,17 , and 18 were done in order to gauge the extent to which the cellular response deteriorated over this period of continuous stimulation. A total of 47 repeat measurements showed a mean final response $84 \%$ that at the outset; this deterioration was sufficiently small that alternating the sequence of measurements for successive cells was judged adequate for compensation.

Signals were led from recording electrodes, via a chlorided silver wire, to a 52K operational amplifier (Analog Devices, Norwood, MA) with a gain of $\times 1000$ and a passband of 1 to $280 \mathrm{~Hz}$. The reference electrode was a spiral of chlorided silver wire placed in the bath. When responses were to be averaged, the amplifier's output signals were amplified an additional $\times 10$, stored with an FM instrumentation recorder (model 3968A, HewlettPackard, Palo Alto, CA), and processed with a PDP-1134 digital computer (Digital Equipment Corp., Marlboro, MA). In most experiments, however, the response was detected with a lock-in analyzer (model 5204, Princeton Applied Research, Princeton, NJ) with a tuned bandpass amplifier $(Q=5)$ set to the stimulus frequency of $25 \mathrm{~Hz}$. The input from the initial amplifier was coupled through a 5- to $100-\mathrm{Hz}$ bandpass filter as well. The lock-in analyzer was used at a full scale deflection setting of $10 \mathrm{mV}$; its output, filtered with a time constant of $1 \mathrm{sec}$, was displayed on a meter and recorded on a chart recorder for subsequent analysis. The dominant noise source in recordings was the thermal noise associated with the recording electrode's resistance which was about $13 \mu \mathrm{V}$ root-mean-square (RMS) in the initial amplifier's passband. The total RMS noise detected by the lock-in amplifier at the stimulus frequency, including about 0.2 $\mu \mathrm{V}$ of pickup of stimulus signals at the largest stimulus amplitudes, was roughly $0.4 \mu \mathrm{V}$. This background was subtracted from all data presented below.

The mechanical stimulus waveform was used as the reference signal for the analyzer. The largest responses were obtained with the analyzer set for a phase lag of $240^{\circ}$ with respect to the stimulus signal. The fact that a positive stimulus-one deflecting the hair bundle in the positive $x$ direction-elicits a negative extracellular response produces a phase lag of $180^{\circ}$. The stimulating circuitry introduces a lag of $23^{\circ}$ in probe motion, while the filters in the recording circuitry contribute $15^{\circ}$. Finally, an adaptation process in the transduction apparatus (Eatock et al., 1979), with a time constant of about 16 msec in the present ionic environment (R. A. Eatock, personal communication), contributes a phase advance of the response by about $22^{\circ}$ with respect to the stimulus. The experimentally measured phase lag is thus in reasonable agreement with the expected value of $196^{\circ}$.

The lock-in analyzer is calibrated to read RMS voltage when presented with sinusoidal input signals. In order to facilitate comparison of the present results with other physiological studies, it was necessary to convert the analyzer output into the equivalent peak-to-peak voltage. For a sinusoidal signal, this would require multiplication by a factor of 2.83 . Since the receptor current is not a pure sine wave, this correction is not sufficient. By graphical analysis of voltage clamp current records (A. J. Hudspeth and D. P. Corey, unpublished observation) and by comparison of the output of the lock-in analyzer with averaged input signals (Fig. 3), the appropriate factor was determined to be 3.38. Calibration of the recording apparatus by application to the amplifier input of a $25-\mathrm{Hz}$, sinusoidal signal $10 \mu \mathrm{V}$ in peak-to-peak amplitude indicated that the amplifiers and filters attenuated signals by $9 \%$; the data presented accordingly represent analyzer output values multiplied by a factor of 3.71 .

Voltage clamp experiments employed two intracellular microelectrodes and recording procedures detailed previously (Corey and Hudspeth, 1979a). For local application of aminoglycosides to hair bundles, microelectrodes with tip diameters of 0.1 to $0.3 \mu \mathrm{m}$ were filled with 400 mM dihydrostreptomycin sulfate (ICN Pharmaceuticals, Cleveland, $\mathrm{OH}$ ) neutralized with $25 \mathrm{~mm} \mathrm{KOH}$. The drug was allowed to diffuse from the electrode tip in the absence of braking current or was ejected iontophoretically by passage of positive currents of 0 to $100 \mathrm{nA}$.

Modeling of responses. Because of the hair bundle's shape and the hair cell's location within an epithelium, the flow of transduction current around a hair cell is undoubtedly of complex geometry. For comparison with experimental measurements, however, the current flow may be approximated as that out of point sources, or into point sinks, located at the site or sites of transduction channels. The current entering the apical surface of a hair cell during an oscillatory stimulus is biphasic; the direction of current flow is alternately outward and inward. Since the inward current flow due to stimulation in the positive direction is severalfold larger than the outward flow during negative stimulus components, the analysis below deals with transduction channels as current sinks.

When a single hair cell is stimulated, transduction current enters the cell's apex and exits through its basolateral surface. The current loops then close by the flow of extracellular current from the cell's base to the site of transduction. In terms of the potentials that it produces in the surrounding medium, the hair cell thus resembles an electrical dipole. Were current $I$ to flow through a medium of a uniform resistivity $\rho$ from a point source at a hair cell's base to a point sink at its apex, the extracellular potential at any point in the surrounding medium would be given by (Attwood, 1941; Plonsey and Collin, 1961):

$$
V_{c}=\frac{I \rho}{4 \pi}\left(\frac{1}{r_{\text {source }}}-\frac{1}{r_{\text {sink }}}\right)
$$

Here $r_{\text {source }}$ and $r_{\text {sink }}$ are, respectively, the distances of the recording site from the source and sink. The potential at a recording site due to multiple sources and sinks then may be obtained, on the basis of superposition, by summing the contributions from each. 
It is obvious that current flow about the base of a hair cell will deviate strongly from the dipole model, particularly because the high resistance of the basolateral membranes of the hair and supporting cells will constrain most current flow within the intercellular spaces. Current flow also will be influenced by the diffusion barrier formed by the tight junctions among hair and supporting cells (D. P. Corey and A. J. Hudspeth, unpublished observation). On the other hand, current entering a sink or sinks at the cellular apex will largely flow through free solution. Distortions in the current flowing about the hair bundle are to be anticipated only where a significant fraction of the volume is occupied by resistive structures, for example at the site of close packing of stereocilia near their distal tips.

For the saline solution employed in these experiments, the resistivity at $22^{\circ} \mathrm{C}$ was measured as $79.9 \mathrm{ohm}-\mathrm{cm}$ (CDM104 conductivity cell and CDM2e meter, Radiometer, Copenhagen, Denmark). In the calculation of model potential distributions, the value of $I$, the peak-to-peak current through all of the transduction channels, was taken as $175 \mathrm{pA}$. Because it cannot pull the hair bundle, a stimulus probe pushed against the shortest stereocilia elicits only about 80 to $85 \%$ of the full response of which a cell is capable. A current of $175 \mathrm{pA}$ accordingly corresponds to a maximal current of about $210 \mathrm{pA}$, a value in very reasonable agreement with that measured in an earlier, voltage clamp study (Corey and Hudspeth, 1979a) and with subsequent observations.

The potential distributions expected at the various recording sites were calculated for five model current distributions: $(A)$ a single sink at the base of the kinocilium, $(B)$ equal sinks at the bases of each of the 50 stereocilia in a typical hair bundle, $(C) 984$ equal sinks uniformly spaced at an interval of $0.32 \mu \mathrm{m}$ along the 50 stereocilia, $(D) 177$ equal sinks spaced at the same interval but confined to the distal portions $(20 \%)$ of the stereocilia, and $(E) 50$ equal sinks at the distal tips of the stereocilia. In all distances, the current source of the dipole was situated approximately at the midpoint of the basolateral membrane area, at coordinates $(-3.0,0.0$, -25.0 ). For each model, the distances of a given recording site from each current source and sink were calculated first from the relevant $x, y$, and $z$ axis coordinates. The potential contributions of source and sink at the recording site then were determined from the formula above and added. Finally, the contributions from all dipolesfrom 1 in case $A$ to 984 in model C-were summed. This procedure then was repeated for the remaining recording sites. Calculations were conducted on a digital computer (model 2647A, Hewlett-Packard, Palo Alto, CA) to a precision of $0.01 \mu \mathrm{m}$ and $0.01 \mu \mathrm{V}$.

Because of the motion of the hair bundle during stimulation, the locations of the sinks in some of the models $(\mathrm{C}, \mathrm{D}$, and $\mathrm{E})$ were not fixed. To approximate the effect of the $1-\mu \mathrm{m}$ deflection of the hair bundle, all calculations were repeated for static offsets of $0.25,0.50$, and $0.75 \mu \mathrm{m}$. The predicted potentials at each recording site then were averaged from these. In no case did the model potential distribution produced by this procedure differ importantly from that expected for a stationary hair bundle in its rest position.

\section{Results}

Validation of recording technique. When a recording electrode is placed near the hair bundle of a cell subjected to continuous mechanical stimulation with a vibrating probe (Fig. $2 A$ ), the electrode detects a potential up to $17 \mu \mathrm{V}$ in peak-to-peak equivalent amplitude with respect to a distant reference electrode (Fig. $2 B$ ). This signal may be separated from background noise of a comparable amplitude either by computer averaging or by use of a lock-in analyzer tuned to the stimulus frequency (Fig. 3). Stimuli directed along the axis of hair bundle symmetry ( $x$ axis) produce an extracellular potential out of phase with the stimulus signal. A positive stimulus, which deflects the hair bundle toward its kinocilium, evokes a negative extracellular potential; conversely, negative stimuli produce positive signals. This polarity is that expected for a potential due to the transduction current. Positive stimuli open ionic channels in the hair cell's membrane (Corey and Hudspeth, 1979a), allowing the flow of current into the cell and thus effecting a depolarization. The flow of this current across the resistivity of the extracellular medium would be expected to make the potential just outside of the hair bundle negative with respect to a distant ground.

That the extracellularly recorded signal is caused by the flow of current associated with the transduction process and is not due to hydrodynamic artifacts or interference from the stimulus signal may be established by several controls. First, even with the recording electrode in place adjacent to the hair bundle and the stimulus probe in motion, no signal is observed until the probe engages and begins to deflect the hair bundle (Fig. $2 B$ ). If the hair bundle is not stimulated, oscillation of the stimulus probe through a peak-to-peak amplitude of $1 \mu \mathrm{m}$ produces no significant signal even when the probe is within $0.5 \mu \mathrm{m}$ of the recording electrode or in contact with it.

Second, the response elicited by $1-\mu \mathrm{m}$ stimuli oriented along the cell's axis of bilateral symmetry ( $x$ axis; Shotwell et al., 1981) is severalfold greater than that due to stimulation along the perpendicular $(y)$ axis (Fig. $2 C$ ). In 17 measurements, the response to perpendicular stimulation was $0.24 \pm 0.03$ (SEM) that to stimulation along the axis of symmetry. This difference is anticipated on the basis of the directional sensitivity of hair cells previously noted in recordings of afferent action potentials (Lowenstein and Wersäll, 1959) and of intracellular responses (Shotwell et al., 1981). The persistence of any response during perpendicular stimulation probably reflects the fact that the rather large stimuli are not directed exactly perpendicularly to the cells' axis of symmetry but have some component of motion along this axis.

A third indication that the extracellularly recorded potential reflects the transduction process is that it may be blocked by ototoxic drugs that also interfere with the receptor potential. Figure $2 D$ shows reduction of the extracellular signal following iontophoretic application of the aminoglycoside antibiotic, dihydrostreptomycin, at concentrations of about $10 \mu \mathrm{M}$. This concentration largely blocks the receptor potential measured by microphonic 

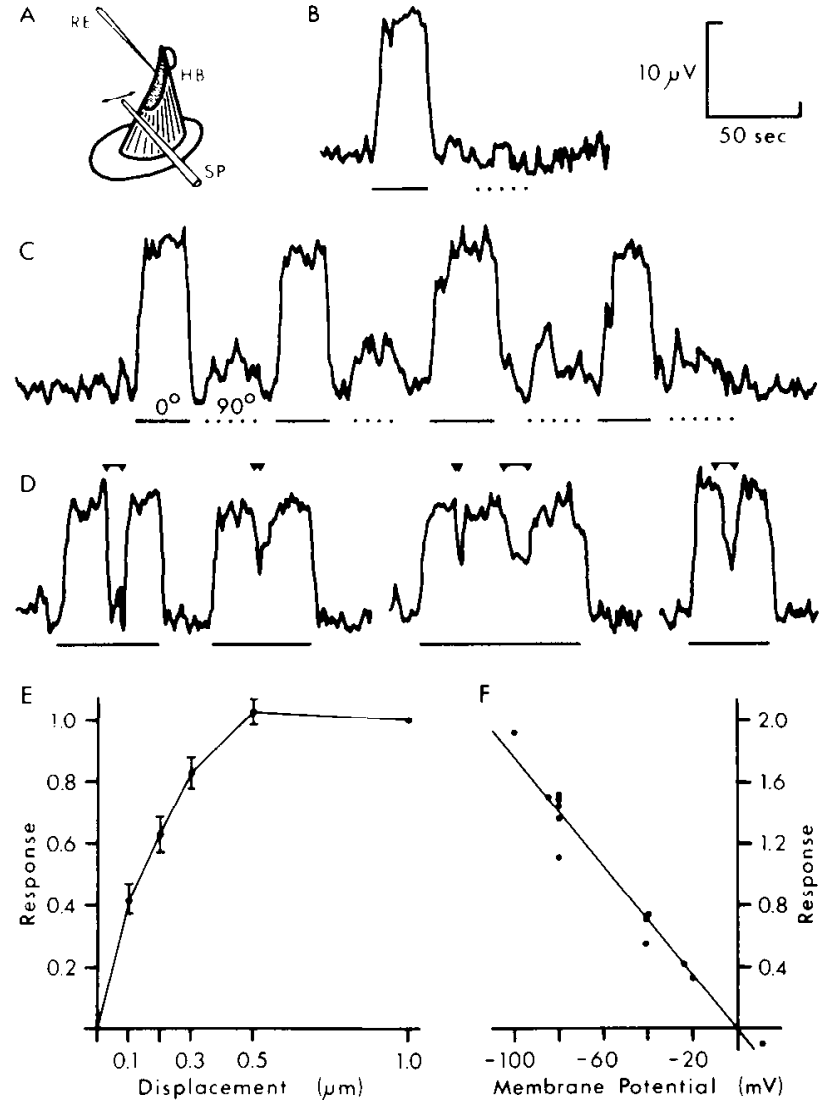

Figure 2. Control experiments indicaling that the extracellularly recorded signals correspond to the flow of transduction current. $A$, Schematic diagram of the experimental arrangement. The stimulus probe $(S P)$ is applied horizontally to the short side of the hair bundle $(H B)$, which is driven through a $25-\mathrm{Hz}$, sinusoidal displacement of $1.0 \mu \mathrm{m}$ as measured at the tip of the kinocilium. The recording electrode $(R E)$ approaches the hair bundle from the side opposite the stimulus probe; accordingly, all measurements are taken from one side of the hair bundle. $B$, Output of the lock-in analyzer with the recording electrode on the top of the hair bundle (position 17) and with the stimulus probe engaging and stimulating the hair bundle (continuous bar) or moving adjacent to it (dotted line). There is no detectable electrical or hydrodynamic artifact from the moving probe when it is not driving the hair bundle. $C, \mathrm{De}-$ pendence of response on stimulus orientation. A recording electrode in position 17 detects a robust response when the hair bundle is stimulated through $1.0 \mu \mathrm{m}$ along the cell's axis of bilateral symmetry $\left(0^{\circ} ;\right.$ solid bar $)$. When oriented perpendicular to this axis $\left(90^{\circ}\right.$; dotted line $)$, a stimulus of equal amplitude yields a much smaller response. $D$, Blockage of response by iontophoretic application of an aminoglycoside antibiotic. In these three records, stimuli are applied to hair bundles as indicated by the continuous bars beneath the records. The symbols above the traces show the durations of iontophoresis of dihydrostreptomycin, whose peak concentration around the hair bundle reaches approximately $10 \mu \mathrm{M} . E$, Dependence of the extracellularly recorded response on stimulus amplitude. All stimuli are oriented along the cell's axis of symmetry. The responses and their standard errors represent data from 23 measurements on three cells, normalized to the response amplitude for each for $1.0-\mu \mathrm{m}$ stimulation. $F$, Effect of membrane potential on extracellularly recorded responses. While continuous, $1.0-\mu \mathrm{m}$ stimuli are applied, the membrane potential is

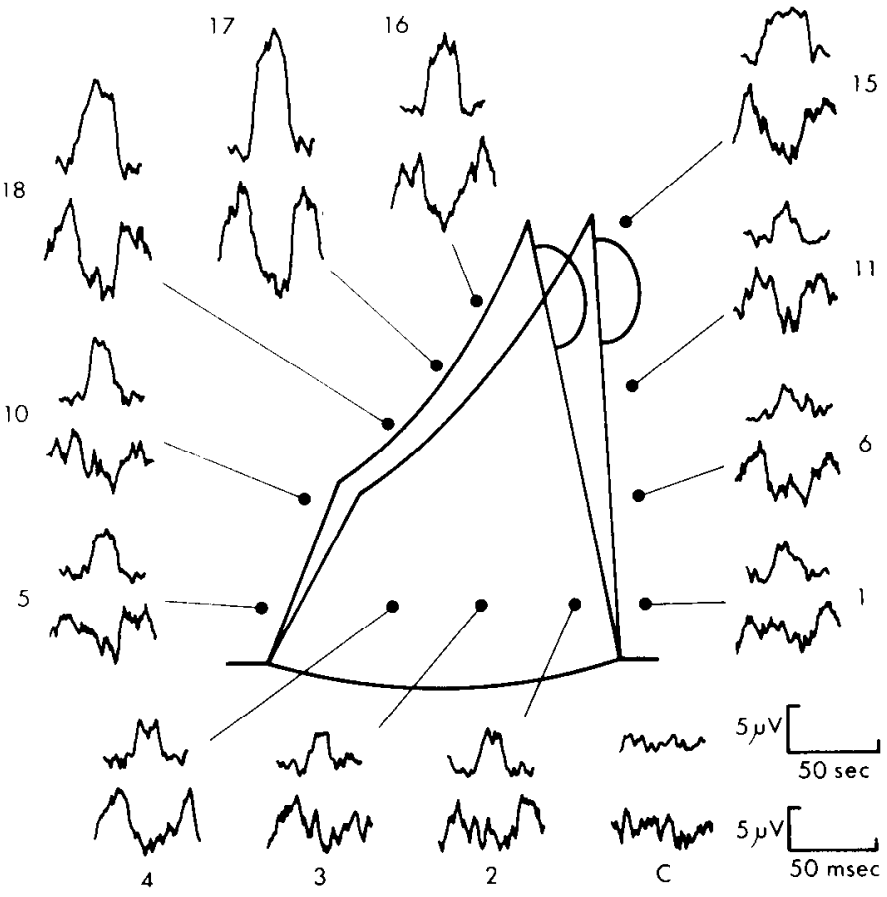

Figure 3. Representative records for 12 recording sites around a hair bundle. The cell is stimulated by driving the hair bundle through $1.0-\mu \mathrm{m}, 25-\mathrm{Hz}$, sinusoidal deflections; the hair bundle is diagrammed at rest and at a deflection to $+1 \mu \mathrm{m}$. The upper record at each recording site is the output of the lock-in analyzer. For comparison, the lower records are computeraveraged extracellular responses from 5 -sec samples of electrode output. The control records $(C)$ show the noise level in each type of trace. Note that responses are consistently larger at the hair bundle's top than at its bottom and that the largest signals occur on the beveled top surface of the hair bundle.

or intracellular recording (Kroese and van den Bercken, 1980; D. Lo and A. J. Hudspeth, unpublished observation).

A fourth feature of the extracellularly recorded signal consistent with its representing a cellular response is its dependence on stimulus amplitude. Like the intracellularly recorded receptor potential (Hudspeth and Corey, 1977), the extracellular signal increases in magnitude for peak-to-peak stimulus amplitudes up to about $0.5 \mu \mathrm{m}$ and saturates for larger stimuli (Fig. $2 E$ ).

Finally, the extracellular potential varies in a predictable manner with changes in the potential across the hair cell's membrane. On the assumption that continuous stimulation of the hair bundle produces a mean conductance $g_{t}$, the mean transduction current $I_{t}$ is given approximately by:

$$
I_{t}=g_{\ell}\left(V_{m}-E_{r}\right)
$$

adjusted to the indicated values with a two-electrode voltage clamp. Data are taken from nine cells and are normalized to the response amplitudes at the initial membrane holding potential of $-60 \mathrm{mV}$. The extracellular recording electrode is in position 17. The negative response during clamping to $13 \mathrm{mV}$ corresponds to a phase reversal of the signal detected with the lock-in analyzer. 
Here, $V_{m}$ is the membrane potential and $E_{r}$ is the reversal potential for the transduction channels, which is about $-2 \mathrm{mV}$ in the present ionic environment (Corey and Hudspeth, 1979a). The expected extracellular potential $V_{c}$ is proportional to the transduction current (see "Materials and Methods"); therefore:

$$
V_{e}=\frac{g_{\varphi} \rho\left(V_{m}-E_{r}\right)}{4 \pi}\left(\frac{1}{r_{\text {source }}}-\frac{1}{r_{\text {sink }}}\right)
$$

where $\rho$ is the resistivity of the medium and $r_{\text {source }}$ and $r_{\text {sink }}$ are the distances of the recording electrode from the source and sink. Figure $2 F$ shows a plot of the magnitude of the extracellular signal against the potential to which the membrane is set by voltage clamping. The linear plot, with its $x$ intercept of $0 \mathrm{mV}$ quite near the reversal potential measured by intracellular voltage clamping (Corey and Hudspeth, 1979a), is in good agreement with the theoretical expectation.

In the light of these findings, it seems reasonable to regard the extracellularly recorded signal as the extracellular receptor potential of an individual hair cell.

Spatial distribution of the extracellular receptor potential. Because the amplitude of the extracellular receptor potential depends inversely on the distance of the recording electrode from the current sinks, measurement of the potential distribution around the hair bundle of a stimulated cell may be used to localize these current sinks. Extracellular receptor potentials at various sites adjacent to the hair bundle, measured both by signal averaging and by lock-in analysis, are shown for a typical cell in Figure 3. It is evident that the responses are smallest about the base of the hair bundle and grow monotonically larger toward the hair bundle's apex. This pattern was found in all of the cells examined. To facilitate comparison of the responses from many hair cells, recordings were made from some or all of 18 specific sites around the hair bundles of each of 22 hair cells (Fig. 1). The results (Table I and Fig. $4 F$ ) confirm the finding of Figure 3; the extracellular receptor potential immediately above the beveled top of the hair bundle is over 4-fold that at the base of the bundle. The responses are of similar amplitudes around the basal circumference of the hair bundle and, in particular, are not exceptionally large near the site of insertion of the kinocilium.

\section{Discussion}

Correlation of results with models. The foregoing results suggest that the principal sink for the flow of transduction-associated current into bullfrog saccular hair cells lies in the distal portion of the hair bundle. An appreciation of the significance of the data may be obtained by comparison of the observed pattern of potentials (Fig. $4 F$ ) with that expected from each of five likely distributions of the transduction elements: at the base of the kinocilium (Fig. $4 A$ ), at the bases of the stereocilia (Fig. $4 B$ ), uniformly along the stereocilia (Fig. $4 C$ ), along the distal fifths of the stereocilia (Fig. $4 D$ ), and at the stereociliary tips (Fig. $4 E$ ). The results evidently exclude some plausible sites for transduction. The shaft of the kinocilium has been shown previously to be unnecessary for transduction in these cells (Hudspeth and Jacobs,

\section{TABLE I}

Potentials measured at various extracellular recording sites during continuous, $25-\mathrm{Hz}$, sinusoidal stimulation through $1.0 \mu \mathrm{m}$

Data have been corrected by a factor of 3.71 to convert lock-in analyzer readings to equivalent peak-to-peak potential values.

\begin{tabular}{|c|c|c|c|c|c|c|}
\hline $\begin{array}{l}\text { Recording } \\
\text { Site }\end{array}$ & $x$ & $y$ & $z$ & Potential & SEM & $\begin{array}{c}\text { Number of } \\
\text { Cells }\end{array}$ \\
\hline & & $\mu m$ & & & & \\
\hline 1 & 0.7 & 0.0 & 1.0 & 2.6 & \pm 0.3 & 22 \\
\hline 2 & -0.6 & -2.9 & 1.0 & 2.6 & \pm 0.2 & 21 \\
\hline 3 & -2.3 & -3.2 & 1.0 & 2.7 & \pm 0.2 & 19 \\
\hline 4 & -3.9 & -3.2 & 1.0 & 2.4 & \pm 0.2 & 20 \\
\hline 5 & -6.3 & 0.0 & 1.0 & 2.8 & \pm 0.2 & 20 \\
\hline 6 & 0.6 & 0.0 & 3.0 & 2.9 & \pm 0.2 & 9 \\
\hline 7 & -0.8 & -2.7 & 3.0 & 3.6 & \pm 0.2 & 9 \\
\hline 8 & -2.2 & -2.9 & 3.0 & 4.0 & \pm 0.2 & 9 \\
\hline 9 & -3.1 & -2.7 & 3.0 & 4.5 & \pm 0.3 & 9 \\
\hline 10 & -5.5 & 0.0 & 3.0 & 4.8 & \pm 0.6 & 9 \\
\hline 11 & 0.5 & 0.0 & 5.0 & 3.6 & \pm 0.2 & 9 \\
\hline 12 & -1.0 & -2.5 & 5.0 & 5.8 & \pm 0.9 & 9 \\
\hline 13 & -2.2 & -2.5 & 5.0 & 5.9 & \pm 0.6 & 9 \\
\hline 14 & -1.2 & -2.3 & 7.0 & 7.6 & \pm 1.1 & 9 \\
\hline 15 & 0.4 & 0.0 & 8.0 & 5.6 & \pm 0.7 & 9 \\
\hline 16 & -2.4 & 0.0 & 6.7 & 9.6 & \pm 0.6 & 14 \\
\hline 17 & -3.0 & 0.0 & 5.4 & 11.3 & \pm 0.6 & 20 \\
\hline 18 & -3.9 & 0.0 & 4.3 & 9.9 & \pm 0.8 & 16 \\
\hline
\end{tabular}

1979); the present findings suggest that the basal body, ciliary necklace, cuticular notch, ciliary rootlets, and adjacent plasmalemma also may be excluded as the transduction elements (Fig. $4 A$ ). Distortion of the apical cellular membranes above or around the cuticular plate is similarly not the event that leads to transduction. The results also largely rule out transduction taking place along the entire lengths of the stereocilia, where stress is distributed (Fig. $4 \mathrm{C}$ ), or at their bases, where flexion occurs (Fig. $4 B$ ). It remains possible, albeit highly unlikely, that the distal ends of stereocilia are constitutively highly permeable and that the ionic current through their tips and along their lengths is modulated by a transduction mechanism at their points of basal insertion.

The spatial resolution of the experimental technique is such that the data are equally consistent with the two remaining models, those positioning sinks along the most distal $20 \%$ of the stereocilia (Fig. $4 D$ ) or at their blunt tips (Fig. $4 E$ ). Transduction could occur in the distal portions of the stereocilia by any of several mechanisms. Bending of individual stereocilia could impose stress on the microfilaments within them, which, in turn, would be communicated mechanically to transduction elements at the site of filamentous insertion on the ciliary tip. Over the restricted range of distance required by the short latency of response in these cells (Corey and Hudspeth, $1979 \mathrm{~b}$ ), a second messenger could diffuse from mechanosensitive sites in the cytoskeleton of each stereocilium to the surface membrane. Another possibility is that the difference in stereociliary length from row to row across the hair cell's apex is essential to transduction (Shotwell et al., 1981). For instance, if, at rest, the tips of the stereocilia were pushed together by forces originating at the cuticular plate, each cilium would be slightly bowed over those in the next shorter row. Displacement of the 

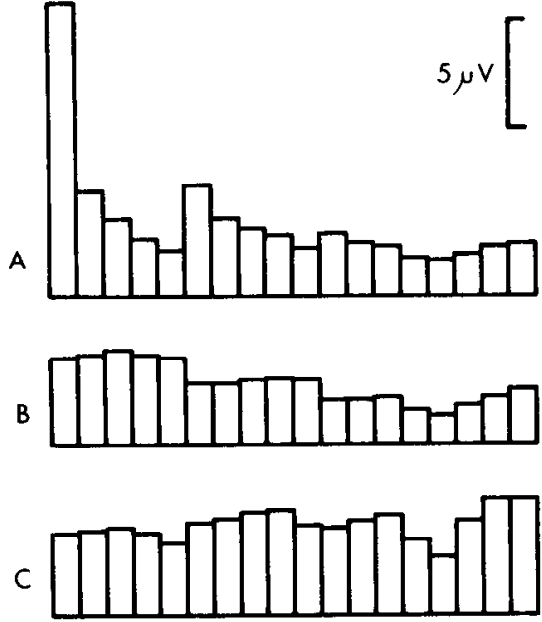

D
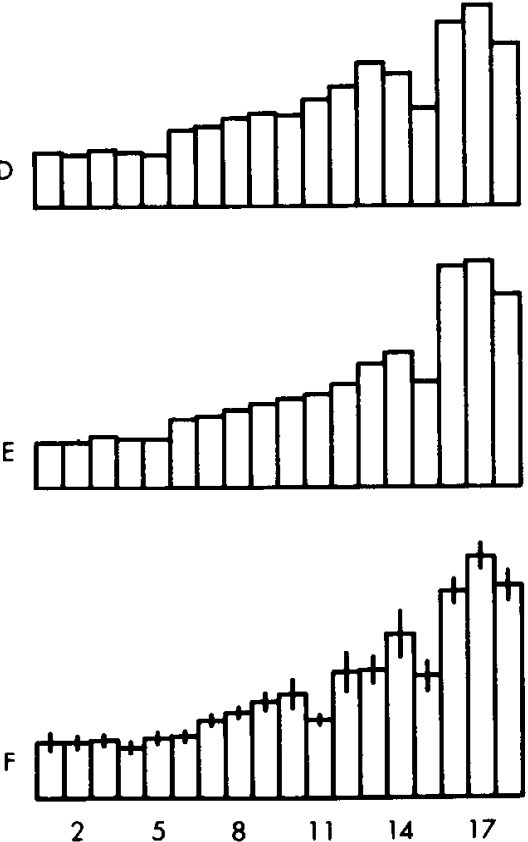

Figure 4. Theoretical and experimental distributions of extracellular potential at the 18 recording sites shown in Figure $1 B$. $A$, The pattern expected if all transduction current flows through channels at the base of the kinocilium. Note the large potential value anticipated at position $1 . R$, Distribution of potentials for 50 identical current sinks situated at the bases of the stereocilia. The model predicts larger signals near the hair bundle's base than at its tip. $C$, Theoretical result for 984 identical transduction sites spaced along the stereocilia at equal intervals of $0.32 \mu \mathrm{m}$. The predicted responses are similar for all of the electrode positions. $D$, Potentials anticipated with 177 equal transduction sinks, at intervals of $0.32 \mu \mathrm{m}$, confined to the distal $20 \%$ of each of the 50 stereocilia. Responses are strikingly larger at the hair bundle's top than at its base. $E$, Predicted distribution of potentials for 50 equal transducers located at the distal tips of the stereocilia. This pattern strongly resembles that of $D$. F , Averaged potential measurements at the 18 recording sites. As indicated in Table I, each bar shows the mean and its standard error for un-normalized data from 9 to 22 different cells. The distribution of potentials, with the largest signals at the top of the hair bundle, is equally consistent with that expected from the sink distributions modeled in $D$ and $E$. hair bundle then might alter the extent of this effect and thus effect transduction at the site of bowing. Separation of the stereocilia upon stimulation in the positive direction might increase the exposure of transduction channels (Malcolm, 1974). Finally, transduction might occur at the sites of contact between adjacent stereocilia due to shear between transduction elements on the apposed membranes.

Consequences of transducer location for signaling. Localization of the transduction channels at the distal ends of the stereocilia must produce some attenuation of the signal reaching the cell body and, thus, in the signal ultimately effecting synaptic transmission from the hair cell to the afferent nerve fiber. The attenuation, however, is probably not severe (Wiederhold, 1976). To a first approximation, a stereocilium may be modeled as a uniform, cylindrical cable terminating in an isopotential cell body of resistance $r_{c b}$ about 500 megohms and of capacitance $c_{c b}$ averaging $28.5 \mathrm{pF}$. The specific resistance $\left(R_{m}\right)$ and specific capacitance $\left(C_{m}\right)$ of the membrane in the stereocilium are assumed to be similar to those of the cell body.

For a stereocilium of diameter $d$ and of internal specific resistivity $R_{i}$, the internal resistance, membrane conductance, and membrane capacitance per unit length are, respectively (Jack et al., 1975, pp. xv-xvi):

$$
\begin{gathered}
r_{i}=\frac{4 R_{i}}{\pi d^{2}} \\
g_{m}=\frac{\pi d}{R_{m}} \\
c_{m}=\pi d C_{m}
\end{gathered}
$$

The behavior of a short, terminated cable excited by harmonic currents may be obtained either by derivation from the cable equations (G. Greene, personal communication) or by replacing values for conductance in published equations with the appropriate complex admittances (Rall and Rinzel, 1973). For sinusoidal signals of angular frequency $\omega$, the membrane admittance per unit of ciliary length is $g_{m}+i \omega c_{m}$. Similarly, the characteristic admittance of the cell body is $g_{c b}+i \omega c_{c b}$, where $g_{c b}=$ $1 / r_{c b}$. The space constant $(\lambda)$ of such a stereocilium is:

$$
\lambda=\frac{1}{\sqrt{r_{i}\left(g_{m}+i \omega c_{m}\right)}}
$$

If a sinusoidal current of amplitude $I$ flows into the distal tip of a stereocilium of length $l$ and if $L=l / \lambda$, the current reaching the cell body $\left(I_{c b}\right)$ may be shown to be (cf. Rall, 1977, equations $3.24,3.26,3.27$, and 3.35 ):

$$
I_{c b}=\mathscr{R}\left[\frac{I e^{i \omega t}}{\cosh L+(1 / B) \sinh L}\right]
$$

The value of $B$, the dimensionless ratio of the cell body's admittance to that of the stereocilium (Rall, 1977, equation 3.26), is:

$$
B=\left(g_{c b}+i \omega c_{c b}\right) \sqrt{\frac{r_{i}}{g_{m}+i \omega c_{m}}}
$$

For saccular hair cells at their resting potential in the saline solution of these experiments, the value of $R_{m}$ is 
approximately 11.9 kilo-ohms-cm ${ }^{2}$, based on the input resistance of 500 megohms (Corey and Hudspeth, 1979a) and an average total cellular surface area of $2372 \mu \mathrm{m}^{2}$. The cellular time constant yields a value for $C_{m}$ of roughly $1.20 \mu \mathrm{F} / \mathrm{cm}^{2}$. Although stereociliary diameter is about 0.2 to $0.3 \mu \mathrm{m}$ in material prepared for transmission or scanning electron microscopy (Hillman, 1972), Nomarski microscopic observations on living cells and computer modeling of the geometrical arrangement of hair bundles both indicate that the stereocilia normally have diameters of about $0.5 \mu \mathrm{m}$. The internal specific resistivity of the microfilament-filled stereocilium might be expected to resemble that of skeletal muscle fibers (Hodgkin and Nakajima, 1972) and, on that basis, is estimated at 150 $\mathrm{ohm}-\mathrm{cm}$ at $22^{\circ} \mathrm{C}$. Based on these data, the value of $\lambda$ is $314 \mu \mathrm{m}$ and that of $B$ is 4.79 for constant currents. Along the longest stereocilia in hair bundles from the bullfrog's sacculus, which measure approximately $8.4 \mu \mathrm{m}$, the attenuation of constant current signals (equation $8, \omega=0$ ) is only $0.6 \%$. For the shortest stereocilia of $3.5 \mu \mathrm{m}$ length, the decrement is $0.2 \%$.

Numerical solution of equation 8 for the same values indicates that attenuation is comparably slight for periodic stimuli of frequencies up to several kilohertz. For the longest stereocilia, the corner frequency at which current is attenuated by $3.01 \mathrm{~dB}$, to $70.7 \%$ its initial value, is $38 \mathrm{kHz}$. The value for the shortest stereocilia is about $220 \mathrm{kHz}$. For numerical values comparable to those in the present model, the cutoff frequency exhibits approximately a direct dependence on the stereociliary diameter and an inverse dependence on the square of ciliary length.

Flow of current into the distal end of the stereocilium represents the most extreme case of attenuation of the signal reaching the cell body. Were the transducers to occur below the stereociliary tips, for example, at the level of the contacts among stereocilia, the signals reaching the cell body would be slightly larger for the identical values of the parameters. Analyses of the attenuation of signals due to injection of current part of the way along a short cable have been presented (Jack and Redman, 1971; Rall and Rinzel, 1973).

A stereocilium is not actually isodiametric along its entire length but characteristically tapers near its basal insertion. For hair cells of the bullfrog's sacculus, stereocilia taper to about half of their diameter over a distance of $1.3 \mu \mathrm{m}$. This constricted region increases the longitudinal resistance of the stereocilium by approximately 20 megohms and further attenuates the transduction signals originating near the stereociliary tip. Quantitatively, however, this effect is not great: when the taper is included in calculations, the steady state current reaching the cell body and the rolloff frequency of the longest stereocilia decrease by less than $1 \%$.

This analysis indicates that, despite their rather small diameters, stereocilia are highly efficient cables in the frequency range employed by the auditory organs of vertebrates. This conclusion rests upon the assumptions that the resistive and capacitative properties of the stereociliary membrane are similar to those elsewhere in the cell and that the internal specific resistivity of the stereociliary core resembles that of cytoplasm. For the last assumption, there is at least indirect evidence: the protein horseradish peroxidase and the dye Lucifer Yellow $\mathrm{CH}$ readily diffuse from the cell body into the stereocilia and kinocilium (A. J. Hudspeth and R. Jacobs, unpublished observations), implying that the ciliary core is at least moderately permeable. The space constant of the stereocilia could be much shorter, and attenuation along their lengths much greater, if such conductance mechanisms as outward rectification (Hudspeth and Corey, 1979a) were concentrated in the stereociliary membrane. This effect would manifest itself, however, only with responses large enough to activate this conductance significantly.

It is apparent that location of the transduction apparatus at or near the distal ends of the stereocilia is by no means precluded by the likely properties of the stereocilia. On the other hand, if the transducers are, in fact, at the stereociliary tips, the upper frequency range over which hair cells respond may be set not only by the speed of operation of the transduction mechanism (Corey and Hudspeth, 1979b) but also by the attenuating properties of stereociliary cables. The systematic shortening of stereocilia in the high frequency portions of the auditory organs of mammals (Lim, 1980) and reptiles (Mulroy, 1974) may represent an accommodation to the attenuating effect of longer cables.

Despite the modest losses of current along the stereocilia, cable properties have a small but significant effect on the sizes of receptor potentials at the tips and bases of the stereocilia. Measured from the distal end, the steady state input resistance of a stereocilium $\left(r_{s c}\right)$ may be shown to be (cf., Jack et al., 1975, equations 7.6 to 7.8 and Rall, 1977, equations 3.26, 3.35, and 3.37):

$$
r_{s c}=r_{c b}\left[\frac{1+B \tanh L}{1+(1 / B) \tanh L}\right]
$$

In the present instance, the input resistance of the longest stereocilium is 561 megohms. Flow of a steady current $I$ into the tip of a stereocilium will produce a local transmembrane potential of $I r_{\mathrm{sc}}$. At the base of the stereocilium, the current will be attenuated as noted in equation 8 above. This current, flowing across the cell's input resistance $r_{c b}$, will produce a potential drop in the cell body given by:

$$
V_{c b}=\frac{I r_{c b}}{\cosh L+(1 / B) \sinh L}
$$

For the present numerical values, the steady state potential change observed in the cell body is $92.1 \%$ that at the stereociliary tip. Current passed from the cell body along the stereocilia will likewise suffer slight attenuation, but estimates of reversal potential under voltage clamp conditions (Corey and Hudspeth, 1979a) should be in error by no more than a few millivolts.

\section{References}

Attwood, S. A. (1941) Electric and Magnetic Fields, pp. 35-42, John Wiley and Sons, London.

Corey, D. P., and A. J. Hudspeth (1979a) Ionic basis of the receptor potential in a vertebrate hair cell. Nature $281: 675-$ 677. 
Corey, D. P., and A. J. Hudspeth (1979b) Response latency of vertebrate hair cells. Biophys. J. 26: 499-506.

Corey, D. P., and A. J. Hudspeth (1980) Mechanical stimulation and micromanipulation with piezoelectric bimorph elements. J. Neurosci. Methods 3: 183-202.

Eatock, R. A., D. P. Corey, and A. J. Hudspeth (1979) Adaptation in a vertebrate hair cell: Stimulus-induced shift of the operating range. Soc. Neurosci. Abstr. 5: 19.

Engström, H., H. W. Ades, and J. E. Hawkins (1962) Structure and functions of the sensory hairs of the inner ear. J. Acoust. Soc. Am. 34: 1356-1363.

Flock, A., B. Flock, and E. Murray (1977) Studies on the sensory hairs of receptor cells in the inner ear. Acta Otolaryngol. (Stockh.) 83: 85-91.

Hillman, D. E. (1972) Observations on morphological features and mechanical properties of the peripheral vestibular receptor system in the frog. Prog. Brain Res. 37: 69-75.

Hodgkin, A. L., and S. Nakajima (1972) The effect of diameter on the electrical constants of frog skeletal muscle fibres. J. Physiol. (Lond.) 221: 105-120.

Hudspeth, A. J., and D. P. Corey (1977) Sensitivity, polarity, and conductance change in the response of vertebrate hair cells to controlled mechanical stimuli. Proc. Natl. Acad. Sci. U. S. A. 74: 2407-2411.

Hudspeth, A. J., and R. Jacobs (1979) Stereocilia mediate transduction in vertebrate hair cells. Proc. Natl. Acad. Sci. U. S. A. 76: 1506-1509.

Jack, J. J. B., and S. J. Redman (1971) An electrical description of the motoneurone, and its application to the analysis of synaptic potentials. J. Physiol. (Lond.) 215: 321-352.

Jack, J. J. B., D. Noble, and R. W. Tsien (1975) Electrical Current Flow in Excitable Cells, Clarendon Press, Oxford.
Kroese, A. B. A., and J. van den Bercken (1980) Dual action of ototoxic antibiotics on sensory hair cells. Nature 283: 395397.

Lim, D. J. (1980) Cochlear anatomy related to cochlear micromechanics. A review. J. Acoust. Soc. Am. 67: 1686-1695.

Lowenstein, O., and J. Wersäll (1959) A functional interpretation of the electron-microscopic structure of the sensory hairs in the cristae of the elasmobranch Raja clavata in terms of directional sensitivity. Nature 184: 1807-1808.

Malcolm, R. (1974) A mechanism by which the hair cells of the inner ear transduce mechanical energy into a modulated train of action potentials. J. Gen. Physiol. 63: 757-772.

Mulroy, M. J. (1974) Cochlear anatomy of the alligator lizard. Brain Behav. Evol. 10: 69-87.

Plonsey, R., and R. E. Collin (1961) Principles and Applica. tions of Electromagnetic Fields, pp. 183-184, McGraw-Hill, New York.

Rall, W. (1977) Core conductor theory and cable properties of neurons. In Handbook of Physiology, E. R. Kandel, ed., Sect. 1, Vol. I, Part 1, pp. 39-97, American Physiological Society, Bethesda, MD.

Rall, W., and J. Rinzel (1973) Branch input resistance and steady attenuation for input to one branch of a dendritic neuron model. Biophys. J. 13: 648-688.

Shotwell, S. L., R. Jacobs, and A. J. Hudspeth (1981) Directional sensitivity of individual vertebrate hair cells to controlled deflection of their hair bundles. Ann. N. Y. Acad. Sci. 374: 1-10.

Wiederhold, M. L. (1976) Mechanosensory transduction in "sensory" and "motile" cilia. Annu. Rev. Biophys. Bioeng. 5: 3962. 